\title{
Parallel hysteroscopic and laparoscopic myomectomy in infertile patients
}

\author{
Béla G. Molnár • Zsolt Kormányos • Virág Márton • \\ Luka Anðelic • Andor Góli
}

Received: 11 August 2011 /Accepted: 16 September 2011 /Published online: 16 October 2011

(C) Springer-Verlag 2011

\begin{abstract}
The past decades have brought about numerous new methods in the treatment of the most frequent benign gynaecological tumour, the myoma, while at the same time, the indications of traditional surgical treatment have also been reassessed. The constant procrastination of pregnancy to older and older age, the frequency of myoma growing by age and the wider application of assisted reproduction techniques have created new conditions for the treatment of myoma. In our research, we analysed the data of 30 infertile patients out of a group of 99 who had hysteroscopic and laparoscopic myomectomy carried out in one session. In the course of 2 years, nine women got pregnant. Besides two miscarriages, the others could carry their pregnancy for at least 34 weeks. Out of the seven deliveries, five (71\%) ended with caesarean section, for obstetrical reasons in all cases. In case of uterus with multiplex myoma, hysteroscopy and laparoscopy can safely be applied in parallel and it does not necessarily imply the termination of the occurring pregnancy by caesarean section.
\end{abstract}

Keywords Fibroid · Infertility · Hysteroscopy · Laparoscopy

\section{Background}

Among infertile patients, the frequency of occurrence of myoma is $2-22 \%$. Although it may seem evident that

B. G. Molnár $(\bowtie) \cdot Z$. Kormányos $\cdot$ V. Márton

Obstetrics and Gynaecology, University of Szeged,

Szeged, Hungary

e-mail: molnargb@gmail.com

L. Anðelic · A. Góli

Obstetrics and Gynaecology, Opšta Bolnica Subotica,

Subotica, Serbia especially submucous myomas may be responsible for infertility, the significance of myomas in infertility is still questioned by many. Undoubtedly in numerous cases, patients with myoma become pregnant spontaneously and carry their pregnancy up to their terminus. However, it unfortunately occurs rarely that one single node deforms the uterus; we are much likely to face uterus with multiplex myoma. In such cases, the elimination of submucous and intramural or subserous nodes in different sessions would result in a loss of time (repeated scheduling, waiting lists) for the infertile patients. Moreover, the patients would be exposed to the risks of anaesthesia and occurrence neopathy for two times. Apparently, in the case of patients suffering from the parallel presence of submucous myoma and intramural or subserous myoma, the elimination of all fibroids is to be realised in one session.

\section{Methods}

In the past 5 years, we have carried out 663 myomectomies, of which 453 were laparotomies, while 163 were hysteroscopic and 161 were laparoscopic eliminations of the fibroid. In 99 cases, the hysteroscopic and laparoscopic enucleation of the myoma node was realised in the course of one single surgical intervention. We hereby disregard the cases in which the intervention had to be transferred into laparotomy. We included those 30 infertile patients in our analysis who, in the course of gynaecological examination, were diagnosed with myoma as well and who underwent parallel hysteroscopic and laparoscopic surgical interventions aiming at eliminating the fibroids. At four of these patients, the lead symptom besides sterility was metrorrhagia. The examination and the surgical treatment of the 
patients at the two institutions were based on identical principles. In order to identify the precise position of the myoma, we used TVS. Of the 30 patients, 15 had already undergone IVF-ET treatment. Twenty-six patients had never been pregnant; one woman had been pregnant once but lost the pregnancy. Abortion occurred in one case among the anamnestic data. Two members of the group had already had deliveries. We had not used hormonal treatment to decrease the size of the myoma, not even in cases where it was larger than $4 \mathrm{~cm}$. We strived for scheduling the interventions for the proliferative period but, due to the waiting lists, operation may have taken place in the secretion phase.

Submucous myomas were eliminated by a monopolar myoma resectoscope (STORZ GmbH, Tuttlingen, Germany and Wisap, Sauerlach, Germany). The splitting of the operation into two sections did not become necessary in either of the cases. When there was a type II fibroid, we applied $5 \mathrm{NE}$ Oxytocin intravenously in order to support the removal of the node, when the resection of the fibroid had already been started.

In the course of the resection of intramural and subserous myomas, we used three (Szeged) and two (Subotica) assistant trocars, respectively. We did not apply vasopressin or hydropreparation during resection. We used monopolar needle only to incise the capsule of the fibroid; the enucleation of the myoma was carried out partly mechanically and, to treat the vessels leading to the lump, partly by a bipolator. The myoma bed was treated, in most of the cases, by serosa-serosa type interrupted stitches (Fig. 1). We had the chance to try the Bearded stitch sewing material as well with which we could sew the myoma bed positioned on the anterior wall, on the bladder base. Should the myoma be of a diameter larger than $4 \mathrm{~cm}$ and of totally intramural position, its bed was sewed in two layers. The enucleated fibroid was eliminated by an electric morcellator (STORZ GmbH, Tuttlingen, Germany and Wisap, Sauerlach, Germany) and was sent for histological examination.

\section{Findings}

The average age of patients was 34.8 years (min., 24 years; max., 42 years). Parallel hysteroscopic and laparoscopic myomectomy was carried out on 30 patients. For 13 patients one myoma was removed by hysteroscopy and one by laparoscopy. For the other 17 patients, more than one myomas were removed either through hysteroscopy or laparoscopy. An overall number of 78 myomas were removed from the 30 patients. Four was the largest number of fibroids removed from one single patient. The average size of submucous myomas was $26 \mathrm{~mm}$ (min., $10 \mathrm{~mm}$; max., $50 \mathrm{~mm}$ ); the average size of intramural nodes was $38 \mathrm{~mm}$ (min., $10 \mathrm{~mm}$; max., $100 \mathrm{~mm}$ ); the average size of subserosal fibroids was $35 \mathrm{~mm}$ (min., $10 \mathrm{~mm}$; max., $50 \mathrm{~mm}$ ). In the course of hysteroscopic myomectomies, the absorption of irrigation fluid remained below $1,500 \mathrm{ml}$ in all of the cases. The average fluid absorption was $350 \mathrm{ml}$ (min., $100 \mathrm{ml}$; max., 1,500 ml). The average operation time was $99 \mathrm{~min}$ ( $\mathrm{min} ., 40 \mathrm{~min}$; max., $210 \mathrm{~min}$ ). Of the whole operation time, hysteroscopy accounted for $25 \%$ and laparoscopy for $75 \%$. Twenty-five patients left the inpatient institution within $24 \mathrm{~h}$ and five patients left on the second day after the operation.

In the course of hysteroscopic interventions, perforation or fluid overload did not occur in either of the cases. In the course of laparoscopic myomectomies, the cave did not open once. Within 1 year, nine women became pregnant. Outside two miscarriages, the others were able to carry their pregnancy for at least 34 weeks. Of the seven deliveries, five (71\%) ended with caesarean section. The reason for caesarean section was obstetrical in all of the cases. During the caesarean sections, we did not meet significant adhesions.

\section{Discussion}

When hysteroscopic and laparoscopic interventions are carried out in one session, we should start with the

Fig. 1 Serosa-serosa type stitches to close the nest fibroid

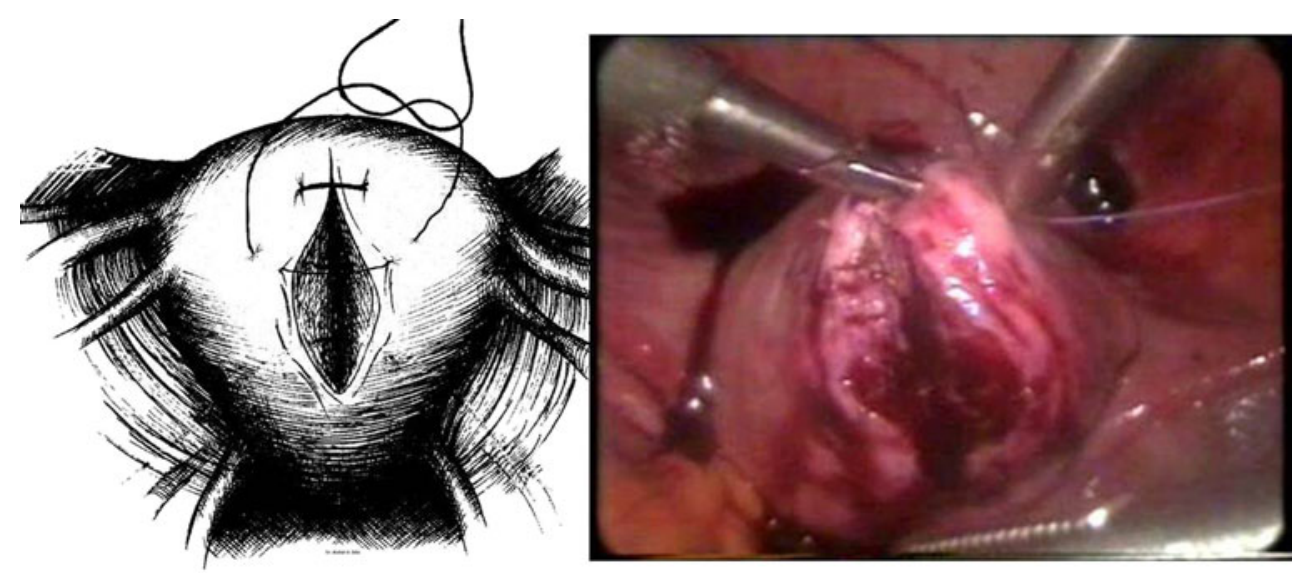


hysteroscopic intervention for practical reasons: this way the justification of diagnosis of perforation, its treatment, the suction of the distending fluid from the abdominal cavity and the impediment of its absorption from the peritoneum become possible. In the case of infertile patients, the diameter of the resectoscope should be the smallest possible [1], in order to minimise the dilatation of the cervix, by which the risk of cervix incompetence at future possible pregnancy is also minimised.

At pregnancies proceeding expanded myomectomies, the question whether the woman can be led into vaginal delivery is always raised. According to our experience, as long as no perforation occurred in the course of the hysteroscopic intervention and the uterine cavity did not open during laparoscopic myoma enucleation, that is, the patient suffered no injury affecting the wall of the uterus in the whole of its thickness; caesarean section is not to be applied as a routine. Unharmed myometrium provides sufficient flexibility and resistance in the course of advancing pregnancy. In the first and second stage of labour, intensified monitoring and the frequent control of the sensitivity of the uterine scar is necessary. Should the uterus become increasingly sensitive to pressure, caesarean section is to be applied. Although theoretically not justified, we do not apply Oxytocin infusion at deliveries following myomectomy for generating or intensifying contractions, similarly to our practice pursued at preceding caesarean sections.

In line with the findings of other work teams [2-4], we did not experience uterus rupture in our patient sample. There have been publications on uterus rupture following myomectomy, but in all of these cases, forced intensification of contractions appeared in the anamnesis [5].

If the myomas are found on the same side, e.g. on the anterior wall of the uterus, the risk of perforation of the uterine wall arises at parallel elimination. The theoretical possibility for this advancement does exist but we have not met any such case in our practice. The perforation of the uterus is rare in the course of hysteroscopic myomectomies; its possibility based on literature findings is ca. 1-2\% [6]. Regarding laparoscopic myomectomies, the opening of the uterine cavity is experienced more often. If we strictly constrain ourselves to stay within the so-called "fake skin" of the myoma while enucleating it, the risk of the opening of the cavity can practically be reduced to zero. Should the cave open even so, it is advisable to close the myoma bed in two layers and provide antibiotic treatment in the postoperative period. This circumstance does not affect the time of hospitalisation [7].

There is no consensus on when a woman may become pregnant after myomectomy [8]. As for us, we share the view that at least 3 months (that is, at least two terminated menstrual cycle) should pass before starting the aspiration for pregnancy. This amount of time is sufficient for the endometrium to regenerate so that, even if conception takes place immediately, there are no more obstacles in front of the fertilised ovum to imbed. Expectation time is not affected by the size of the eliminated myoma; it is rather the size of the wound created during elimination that may act as an influencing factor. Consequently, in cases of hysteroscopic eliminations of large size type I and type II myomas and, for laparoscopic myomectomies, after eliminating myomas positioned mainly intramurally, longer recovery period is expected. In such cases we recommend to wait for 6 months. On the other hand, in our patient sample, one woman became pregnant 3 months after the enucleation of a 4-cm submucous and a 6-cm subserosal-intramural myoma and delivery occurred by a caesarean section on the 38th week. One year after delivery, she repeatedly became pregnant spontaneously; at present she is in the 30th week of her pregnancy.

Proceeding endoscopic myomectomies, provided that neither perforation (hysteroscopy), nor the opening of the uterine cavity (laparoscopy) occurred, there is no obstacle in front of vaginal delivery. However, the majority of these pregnancies are terminated by a caesarean section [9]. Besides obstetrical reasons, the indication for caesarean section is underpinned by previous operation on the active section of the uterus, the striving for safety and the fact that these patients tend to arrive at delivery following a multiannual infertility treatment so they are regarded as high-risk patients. It is important to highlight the difference between the uterus of patients with preceding caesarean section and those undergone myomectomy. In the course of caesarean section, we incise the uterus on the passive part which does not get under tension before, at the end of the next pregnancy, labour begins and contractions become regular. On the contrary, following myomectomy, the uterine wound is practically constantly under tension as pregnancy advances. That is why, in the latter case, increased awareness in pregnancy attendance is by all means justified and, at delivery, the sensitivity of the scar or the explicit extenuation of the scar implies caesarean section [10].

In the course of deliveries led by caesarean section, we did not find significant adhesions due to previous myomectomies. We claim that this circumstance is deducible to the fact that, in the course of laparoscopic myomectomy, we used a sewing technique (serosa-serosa type stitches) which, after application, results in the full covering of the wound by the uterine serosa and only minimal sewing substance runs on the surface of the uterus. This observation is underpinned by the fact that, following our 161 laparoscopic myomectomies, we have not found expressive adhesion formation at those, originally not infertile patients either who later underwent laparotomy due to other reasons (e.g. new myoma formation, ovarian tumour). On the other hand, in the 
course of myomectomies carried out through laparotomy, expressive adhesions had occurred with higher frequency. Therefore, based on our positive experience gained in respect of serosa-serosa type stitches, we have lately turned to applying this sewing technique in laparotomy as well. In order to explain whether less frequent adhesion formation is deducible to laparoscopic intervention or the application of serosa-serosa type stitches, we need to conclude further examinations. Other studies have also come to the conclusion that de novo adhesion formation is deducible to surgery duration, blood loss, number and size of incisions, suturing and number of knots [11].

It may occur that, in the course of caesarean section, we still find myoma on the uterine wall which brings up a further problem of how the myoma should be treated during the intervention. A widely accepted view implies that, during caesarean section, only pedunculated myomas are allowed to be removed as the resection of intramural myomas may result in profuse bleeding and, eventually, in losing the uterus [12]. However, there are tracks of successful myomectomies carried out during caesarean section where the myoma bed was secured by a previously inlaid purse string stitch [13]. Another solution of ligating the uterine arteries parallel to caesarean section in order to decrease post-operative bleeding has also been published [14]. As for ourselves, we pursue the practice of not eliminating the myomas during caesarean section, provided their position does not disturb the sewing of the uterine wound, but we recommend eliminating them in another session, should they cause complains or symptoms.

The deformation of the uterine cave following the hysteroscopic elimination of submucous myomas or the formed scar tissue following the elimination of type II fibroids may become an impediment to the realisation or carriage of planned pregnancy [15]. Proceeding interventions affecting the uterine cave, we provide oestrogen for a month which, by the rapid thickening of the endometrium, decreases the risk of adhesion formation. The placement of mechanical instrument (Folley-catheter, IUD) after intervention may, according to our experience, lead to adhesion formation so it rather worsens than improves the status of the uterine cave.

\section{Conclusion}

In the case of multiplex myomas causing infertility, the parallel hysteroscopic and laparoscopic elimination of myomas is technically possible and does not necessarily imply that future pregnancy can only be terminated by caesarean section. However, taking into consideration that, in this patient group, persistent infertility or previous unsuccessful pregnancies are often included among anamnestic data, the majority of deliveries are carried out through caesarean section.

Acknowledgements The analysis (including the purchase of a few equipment) was carried out in the framework of the project No. HUSRB/0901/214/082 co-financed by the European Union under the Hungary-Serbia IPA Cross-Border Cooperation Programme 2007 2013. The drawing part of the figure was made by Dr. József Imre.

Conflicts of interests The authors report no conflicts of interest. The authors alone are responsible for the content and writing of the paper.

\section{References}

1. Papalampros P, Gambadauro P, Papadopoulos N, Polyzos D, Chapman L, Magos A (2009) The mini-resectoscope: a new instrument for office hysteroscopic surgery. Acta Obstet Gynecol Scand 88(2):227-230

2. Campo S, Campo V, Gambadauro P (2003) Reproductive outcome after laparoscopic or abdominal myomectomy. Eur J Obstet Gynecol Reprod Biol 110:215-219

3. Soriano D, Dessolle L, Poncelet C, Benifla JL, Madelenat P, Darai E (2003) Pregnancy outcome after laparoscopic and laparoconverted myomectomy. Eur J Obstet Gynecol Reprod Biol 108:194-198

4. Landi S, Fiaccavento A, Zaccoletti R, Barbieri F, Syed R, Minelli L (2003) Pregnancy outcomes and deliveries after laparoscopic myomectomy. J Am Assoc Gynecol Laparosc 10(2):177-181

5. Lieng M, Istre O, Langebrekke A (2004) Uterine rupture after laparoscopic myomectomy. J Am Assoc Gynecol Laparosc 11 (1):92-93

6. Mettler L, Wendland EM, Patel P, Caballero R, Schollmeyer T (2002) Hysteroscopy: an analysis of 2-years' experience. JSLS 6 (3):195-197

7. Seracchioli R, Colombo FM, Bagnoli A, Govoni F, Missiroli S, Venturoli S (2003) Laparoscopic myomectomy for fibroids penetrating the uterine cavity: is it a safe procedure? BJOG 110 (3):236-240

8. Kucera E, Mandys F, Drahonovský J, Martin D, Procházka B (2009) Reproductive outcome after laparoscopic myomectomyretrospective analysis 1994-2007. Ceska Gynekol 74(6):431-436

9. Seracchioli R, Manuzzi L, Vianello F, Gualerzi B, Savelli L, Paradisi R, Venturoli S (2006) Obstetric and delivery outcome of pregnancies achieved after laparoscopic myomectomy. Fertil Steril 86(1):159-165

10. Cheng YW, Eden KB, Marshall N, Pereira L, Caughey AB, Guise JM (2011) Delivery after prior caesarean: maternal morbidity and mortality. Clin Perinatol 38(2):297-309

11. Trew G, Pistofidis G, Pados G, Lower A, Mettler L, Wallwiener D et al (2011) Gynaecological endoscopic evaluation of $4 \%$ icodextrin solution: a European, multicentre, double-blind, randomized study of the efficacy and safety in the reduction of de novo adhesions after laparoscopic gynaecological surgery. Hum Reprod 26(8):2015-2027

12. Roman AS, Tabsh KM (2004) Myomectomy at time of cesarean delivery: a retrospective cohort study. BMC Pregnancy Childbirth 4(1): 14

13. Lee JH, Cho DH (2011) Myomectomy using purse-string suture during cesarean section. Arch Gynecol Obstet Suppl 1:35-37

14. Liu WM, Wang PH, Tang WL, Wang IT, Tzeng CR (2006) Uterine artery ligation for treatment of pregnant women with uterine leiomyomas who are undergoing cesarean section. Fertil Steril 86(2):423-428

15. Deans R, Abbott J (2010) Review of intrauterine adhesions. J Minim Invasive Gynecol 17(5):555-569 